ARTICLE

\title{
Magnetic pumping model for energizing superthermal particles applied to observations of the Earth's bow shock
}

\author{
E. Lichko (i) ${ }^{1,2 \bowtie} \&$ J. Egedal ${ }^{1}$
}

Energetic particle generation is an important component of a variety of astrophysical systems, from seed particle generation in shocks to the heating of the solar wind. It has been shown that magnetic pumping is an efficient mechanism for heating thermal particles, using the largest-scale magnetic fluctuations. Here we show that when magnetic pumping is extended to a spatially-varying magnetic flux tube, magnetic trapping of superthermal particles renders pumping an effective energization method for particles moving faster than the speed of the waves and naturally generates power-law distributions. We validated the theory by spacecraft observations of the strong, compressional magnetic fluctuations near the Earth's bow shock from the Magnetospheric Multiscale mission. Given the ubiquity of magnetic fluctuations in different astrophysical systems, this mechanism has the potential to be transformative to our understanding of how the most energetic particles in the universe are generated.

\footnotetext{
${ }^{1}$ Department of Physics, University of Wisconsin-Madison, Madison, WI, USA. ${ }^{2}$ Lunar and Planetary Laboratory, University of Arizona, Tucson, AZ, USA.

凶email: lichko@wisc.edu
} 
S uperthermal populations of ions and electrons are abundant in a wide variety of astrophysical systems throughout the universe, often with distributions characterized by energetic power-law tails ${ }^{1,2}$. However, the consensus on the physical mechanisms that energize these particles is far from settled. While it is well known that plasma can be energized by waves, most theories of wave-particle energization (excluding shocks and magnetic reconnection) are only effective for energizing particles moving at velocities close to the phase velocity of the waves ${ }^{3-5}$. We here present an analysis of particle energization by magnetic pumping. While previous work suggests that this mechanism is only effective up to the phase velocity of the wave ${ }^{6}$, we show that the addition of magnetic trapping of particle orbits renders pumping effective for heating particles moving far faster than the wave speed, an example of which is shown in Fig. 1. This mathematical treatment reveals the underlying Fermi heating mechanism consistent with the formation of energetic power-law distributions.

Diffusive shock acceleration (DSA) ${ }^{7}$ is a classic example of such a Fermi heating mechanism. Given an initial seed population, DSA yields power-law distributions of often relativistic electrons with Larmor radii larger or comparable to the width of the shock front. In this Letter we find that magnetic pumping in the fluctuations generated in the vicinity of Earth's bow shock can provide a seed population of pre-energized magnetized electrons. Thus, along with mechanisms such as stochastic shock drift acceleration (SSDA) ${ }^{8-10}$, magnetic pumping may help address the injection problem of DSA ${ }^{11,12}$

Magnetic pumping is directly related to the pressure anisotropy that naturally forms when a plasma and its magnetic field are compressed ${ }^{13}$. This anisotropy can be moderated by an effective scattering frequency, $v$, caused by processes such as pitch-anglemixing by Whistler waves ${ }^{14}$, or a limited confinement time of the electrons in the magnetic wells, yielding a phase delay between the perpendicular pressure, $p_{\perp}$, and the flow perpendicular to the magnetic field, $\mathbf{u}_{\perp}$. Given this phase delay, when averaged over a pump cycle, mechanical work by $p_{\perp} \nabla_{\perp} \cdot \mathbf{u}_{\perp}$ then becomes finite and is the source of the energy for the magnetic pumping process. In this process energy is transferred from magnetic fluctuations to directly heat the plasma, bypassing the turbulent cascade ${ }^{15-17}$.

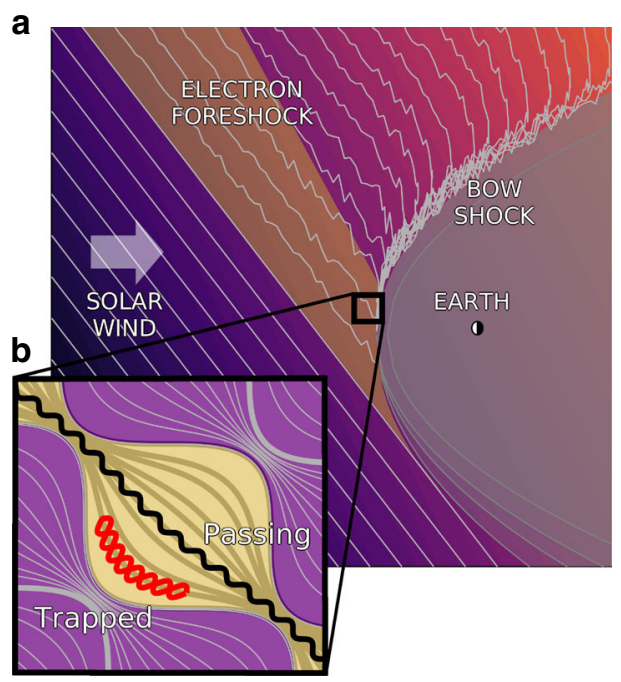

Fig. 1 Cartoon of flux tube with spatial variation. a Cartoon of the incoming solar wind, including the pre-bow shock magnetic fluctuations, similar to the cartoon in Tsurutani and Rodriguez ${ }^{34}$. b The inset shows an example flux tube and a set of trapped (red) and passing (black) particle orbits.
Extensive prior work, however, suggests that magnetic pumping is not effective for energizing superthermal particles. For example magnetic pumping by compressional waves, related to transit-time damping ${ }^{18}$, is shown to be a Landau damping process, where heating is limited to particles moving at the magneticfield-aligned phase velocity of the wave considered, $v_{\mathrm{p}}=\omega / k_{\|}$, where $\omega$ is the angular frequency of the wave and $k_{\|}$is the wavenumber in the direction parallel to the magnetic field ${ }^{19}$. In the framework of quasilinear theory Landau damping causes velocity diffusion limited to particles near the resonance velocity, $v \sim \omega / k_{\|}$, and is derived based on the standard procedure of integrating the plasma kinetic equations along unperturbed particle trajectories. In contrast, here we consider a standing wave geometry and apply the fast transit-time limit ${ }^{20}$ to retain anisotropic effects related to the full electron orbit motion.

Here we show that a quasilinear analysis with trapped electron effects and $v \gg \omega / k_{\|}$yields a velocity diffusion equation similar to that obtained by Lichko $2017^{6}$ for the opposite limit, $v_{\|} \ll \omega / k_{\|}$. More specifically, the slowly-varying background distribution $f_{0}$ is governed by a diffusion equation of the form

$$
\frac{\partial f_{0}}{\partial t}=\frac{1}{v^{2}} \frac{\partial}{\partial v}\left(v^{2} D \frac{\partial f_{0}}{\partial v}\right), \quad D=\omega v^{2} \mathcal{G}\left(\frac{\delta B}{B_{0}}, \frac{\nu}{\omega}\right)
$$

where $\mathcal{G}$ is independent of $v$, but is a function of $v / \omega$, and the magnitude of the magnetic perturbations relative to the background magnetic field, $\delta B / B_{0}$. The result that $D \propto \omega v^{2}$ is evidence of a Fermi heating process with a diffusive step size proportional to the velocity, $\Delta v \propto v$.

\section{Results}

Model of electron trapping. Below we will outline how Eq. (1) is obtained and provide an evaluation of $\mathcal{G}$, a metric of the effectiveness of the pumping process. Meanwhile, we will compare these predictions to observations by the Magnetospheric Multiscale (MMS) mission in the region of the Earth's bow shock ${ }^{21}$. Within the electron foreshock there are ripples, variations in the magnetic field itself, that have been shown to be a source of electron acceleration, as well as other large-amplitude magneticfield fluctuations ${ }^{22-26}$, an example of which was recorded on October 7 th, $2015^{27}$. From the time histories of $|B|, n, T_{\mathrm{obs}}$, and $T_{\text {obs }} / T_{\text {adiabatic }}$ as shown in Fig. $2 \mathrm{a}-\mathrm{d}$, the temperature increase is greater than would be expected from compressional heating alone. The evolving pitch-angle-averaged distribution functions in the foreshock are shown in Fig. 2e for the times marked in Figs. 2a-d. These distribution functions demonstrate energy transfer consistent with a Fermi heating mechanism, where $\Delta v \propto v$, which in log-log format can be seen in the shift of the energetic power-law tails at a constant slope.

We first aim to demonstrate that the observed anisotropy is consistent with electron trapping in a standing wave perturbation and is representative of the perturbed distribution function driven during each pumping cycle. We consider the limit where the bounce time, $\tau_{\mathrm{b}}$, is much smaller than the time scales associated with the waves. The instantaneous particle orbits are then described by the magnetic moment, $\mu=m v_{\perp}^{2} /(2 B)$ and the total energy $U=\mathcal{E}-e \Phi$, where $\mathcal{E}=\frac{1}{2} m v^{2}, e$ is the positive electron charge, and $\Phi$ is the electrostatic potential. We assume electron energies larger than the electrostatic potential associated with the perturbations ${ }^{28}$ such that the $\mathbf{v} \times \mathbf{B}$ part of the Lorentz force dominates the orbit motion. Using the additional assumption that electrons are well-magnetized, with the Larmor radius smaller than the perpendicular wave length of the perturbations, $\rho_{\mathrm{L}}<\lambda_{\perp}$, for the parameters in this bow shock crossing the model is appropriate for energies $100 \mathrm{eV} \lesssim \mathcal{E} \lesssim 100 \mathrm{keV}$. 

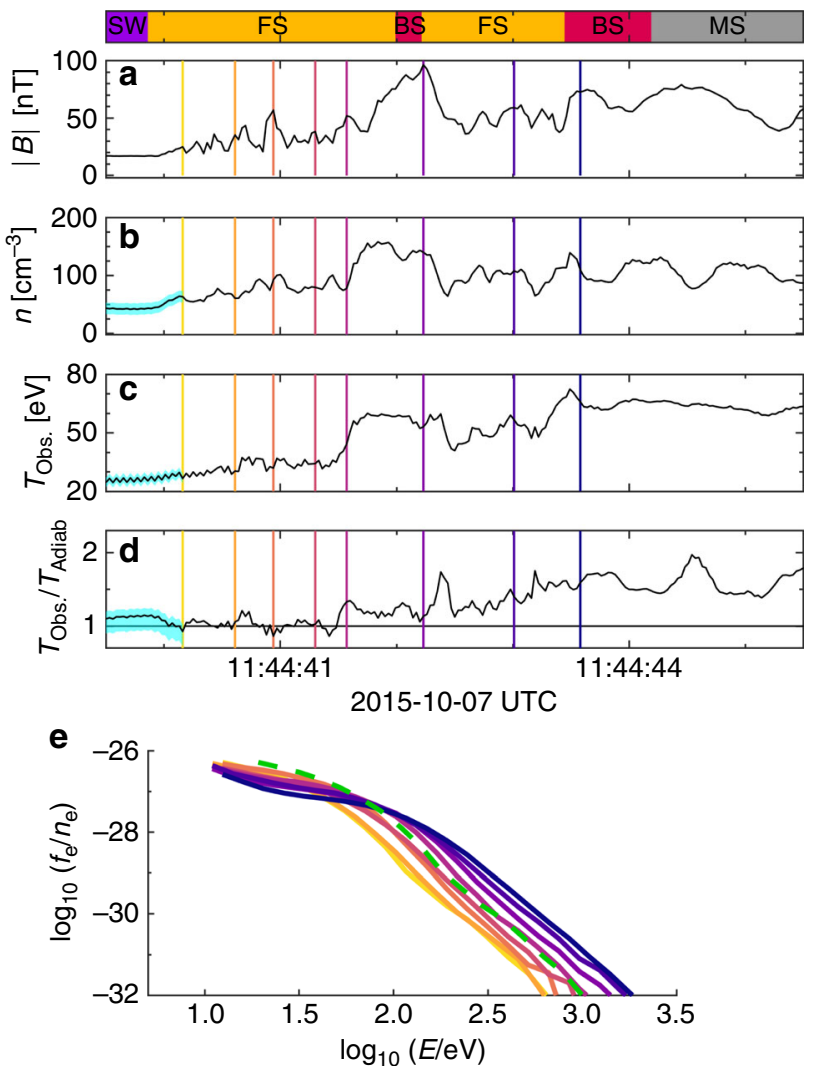

Fig. 2 MMS data for bow shock crossing. a Plot of the average magnetic field from MMS1 as a function of time. The bar on the top of the plots denotes the part of the shock that the spacecraft is observing. The spacecraft is initially in the solar wind (SW) approaching the Earth's bow shock, then travels through the foreshock (FS) and the bow shock (BS) before entering the Earth's magnetosheath (MS). The part of the plot highlighted in light blue denotes the low density of the solar wind. This low density affects spacecraft performance, leading to uncertainty in the measurement of $T_{\text {Obs. }} / T_{\text {Adiab. in }}$ this region. $\mathbf{b}$ Plot of the density from MMS1 as a function of time. c Plot of the observed temperature $\left(T_{\text {Obs. }}=\operatorname{Tr}\right.$ $(P) /(3 n))$ from MMS1 as a function of time. $\mathbf{d}$ Plot of the observed temperature as compared to the temperature expected from compressional heating alone $\left(T_{\text {Adiab. }}=n^{2 / 3}\right)$. e Pitch-angle-averaged distributions at the times denoted by the colored lines in a-d. The green dashed line denotes what the final distribution at the last point should look like if it only underwent compressional heating from the initial yellow time point to the final blue time point. All of these time points are chosen to be near peaks of the magnetic fluctuations spaced upstream of this quasi-perpendicular shock front, $\theta_{B n}=83^{\circ}$, at Alfv́nic Mach number, $M_{A}=6.3^{27}$.

At each time point during the magnetic pump cycle an instantaneous electron orbit is fully characterized by $\mu$ and $\mathcal{E}$. In turn, from the multiple time scale method ${ }^{20,29}$ the distribution must be constant along these instantaneous orbits, reducing the dimensionality of the problem.

Starting with the drift kinetic equation ${ }^{30}$ with pitch-angle mixing, i.e.,

$$
\frac{d f}{d t}=\nu \mathcal{L} f, \mathcal{L}=\frac{\partial}{\partial \xi}\left(1-\xi^{2}\right) \frac{\partial}{\partial \xi}, \xi=\frac{v_{\|}}{\left(v_{\|}^{2}+v_{\perp}^{2}\right)^{1 / 2}},
$$

we change variables from $f\left(t, x, v_{\perp}, v_{\|}\right)=f(t, \mathcal{E}, \chi)$, where $\chi=\Lambda$ / $\left(j^{2}+\Lambda\right), \Lambda=\mu B_{0} / \mathcal{E}$, and $j=J /(4 v L)=1 /(v L) \int_{0}^{L_{\mathrm{b}}} v_{\|} d x$ with $L_{\mathrm{b}}$ denoting the bounce point and $L$ the length of the flux-tube element. Here $\mathcal{E}$ and $\chi$ are both constant of motion variables where $\chi$ is representative of $v_{\perp}^{2} / v^{2}$ along the instantaneous orbits.
Using these new variables and following the approach of Montag et al. ${ }^{31}$ and Egedal et al..$^{32}$, we obtain an orbit-averaged form of Eq. (2)

$$
\frac{\partial f}{\partial t}-\left.H(t, \chi) \mathcal{E} \frac{\partial f}{\partial \mathcal{E}}\right|_{\chi, t}=\nu\langle\mathcal{L}\rangle_{x} f
$$

where $H=\left.(2 / j)(\partial j / \partial t)\right|_{\chi}$, and the orbit average $\langle(\ldots)\rangle_{x}$ is defined in the Methods subsection Orbit averaging of the Lorentz operator.

Electron trapping in spacecraft data. We solve Eq. (3) numerically, assuming an initial isotropic distribution and a standing wave magnetic field, $\tilde{B}(x, t) \equiv B(x, t) / B_{0}=1-\left(\delta B / B_{0}\right) \sin (\omega t) \cos \left(k_{\|} x\right)$. The resultant distribution functions are shown in Fig. $3 \mathrm{f}-\mathrm{i}$ for selected positions along the flux tube at a time $t_{0}$ with a representative amplitude $\sin \left(\omega t_{0}\right)\left(\delta B / B_{0}\right)=0.5$.

Despite the idealized form of the magnetic perturbation, there is good agreement between these model distribution functions and the distribution functions observed by MMS, as shown in Fig. 3a-d, over the course of a single fluctuation of commensurate size, as shown in Fig. 3e. As documented further in the Method subsection Observations of distributions throughout the encounter, anisotropic distributions of this form are observed on all four MMS spacecraft throughout the event. To emphasize the need for pitch-angle scattering the model distributions in Fig. $3 \mathrm{f}-\mathrm{i}$ were generated for $v=0$ and have sharper features in velocity space compared to those observed by MMS.

Magnetic pumping model. To estimate of the aforementioned effective scattering rate we integrate the model in Eq. (3) for various values of $v$ to match the MMS observations. For example, the distribution displayed in Fig. 3k was obtained by integrating Eq. (3) with $v /(\omega / 2 \pi)=0.75$ and provides a good match to the MMS distribution in Fig. 3d. A more detailed description of this matching process is detailed in the Methods subsection Obtaining an estimate for the effective scattering frequency, as well as ref. ${ }^{33}$.

The agreement demonstrated above suggests that the model is capturing the anisotropic features of the observed distribution functions. While the inferred amount of scattering is too low for SSDA $^{10}$ to be effective, it is near optimal for magnetic pumping and the analysis can now be extended to address the energization of the electrons over many cycles. Following the blueprint of the quasilinear method, we then separate the distribution function into the slowly-varying, isotropic background distribution, $f_{0}$, and the anisotropic portion of the distribution function, $f_{1}$,

$$
f=f_{0}(t, \mathcal{E})+f_{1}(t, \mathcal{E}, \chi), \quad f_{0}(t, \mathcal{E})=\langle f(t, \mathcal{E}, \chi)\rangle_{\chi},
$$

where the pitch-angle averaging $\langle(\ldots)\rangle_{\chi}$ is defined in the Method subsection Particle conservation in $\langle(\ldots)\rangle_{\chi}$. To make Eq. (3) more analytically tractable, the Lorentz operator is approximated with the Krook operator, $\mathcal{L}_{\mathrm{K}}=-C_{\mathrm{K}}\left(f-\langle f\rangle_{\chi}\right)$, where the factor $C_{\mathrm{K}}=$ $1.15 /\left(\delta B / B_{0}\right)^{1.13}$ is derived in the Methods subsection Calibrating the Krook operator as a function of $\delta B / B_{0}$. In addition, the anisotropic part of the distribution function, $f_{1}$, as well as the other relevant anisotropic terms $H(t, \chi)$ and $h(t, \chi)=H-\langle H\rangle_{\chi}$ are Fourier transformed such that $f_{1}=\sum_{n} f_{1}^{n} e^{i n \omega t}, H=\sum_{n} H^{n} e^{i n \omega t}$, and $h=\sum_{n} h^{n} e^{i n \omega t}$.

By inserting these expansions and Eq. (4) into Eq. (3) an equation for the anisotropic part of the distribution function is 

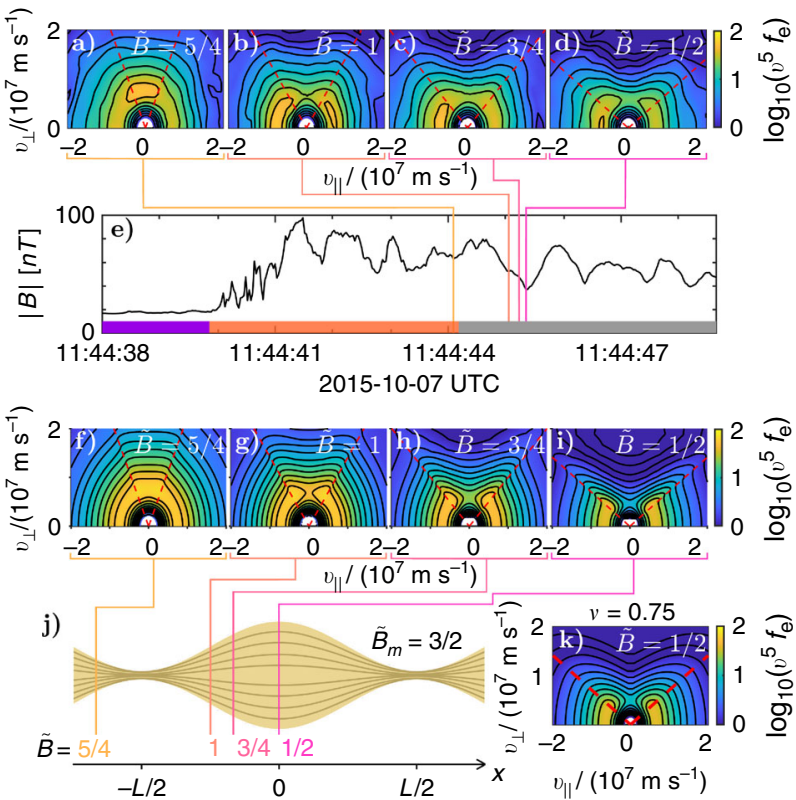

Fig. 3 Comparison of MMS observations with model. a-d Electron distributions recorded by the Fast Plasma Investigation (FPI) instruments on MMS3 at 30 ms resolution for the rippled foreshock event reported in ref. 27 , for the estimated points along the fluctuation where $\tilde{B} \in\{5 / 4$, $1,3 / 4,1 / 2\}$. The distributions are weighted by the factor $v^{5}$ to visually enhance the anisotropic features. The trapped-passing boundary is denoted by a red dashed line. e Magnetic-field strength along the foreshock encounter, where the colored lines denote the times where the distributions in a-d were taken. The purple bar on the bottom denotes the part of the observation that is in the solar wind, followed by an orange bar that denotes the foreshock and bow shock, then a gray bar for the part of the observation that is in the magnetosheath. $\mathbf{f}-\mathbf{i}$ Expected distribution functions computed using Eq. (3) integrated at $\left(\delta B / B_{0}\right) \sin \left(\omega t_{0}\right)=0.5$. The distributions are evaluated at the same $\tilde{B}$ inferred from the MMS data in a-d. In this comparison we have assumed the Taylor hypothesis ${ }^{35}$, that the changes in $B$ recorded by the spacecraft are mainly caused by the spatial, not temporal, variations. For all electron distributions, the red dashed lines indicate the trapped/passing boundaries, characterized by $v_{\perp}^{2} / v_{\|}^{2}=\left(B_{0}+\delta B\right) /$

$B\left(t_{0}, x\right)-1$. Electrons with $\left(v_{\|}, v_{\perp}\right)$ in the vicinity of these boundaries follow orbits which stagnate $\left(v_{\|} \simeq 0\right)$ where $\partial B / \partial t$ is maximal, causing the orbit average of $\partial \mathcal{E} / \partial t=\mu(\partial B / \partial t)$ to be positive. This explains the enhanced values of $f$ along the trapped-passing boundaries. $\mathbf{j}$ shows a cartoon version of the flux tube. $\mathbf{k}$ shows the theoretical distribution in $\mathbf{i}$ scattered with the Lorentz operator, $\mathcal{L}$, for $\nu /(\omega / 2 \pi)=0.75$.

found,

$$
f_{1}^{n}=K_{n} \mathcal{E} \frac{\partial f_{0}}{\partial \mathcal{E}}, \quad K_{n}=\frac{h^{n}\left(-i n \omega+C_{\mathrm{K}} \nu\right)}{n^{2} \omega^{2}+\left(C_{\mathrm{K}} \nu\right)^{2}} .
$$

Inserting $f_{1}$ back into Eq. (3), an evolution equation is obtained for the slowly-varying background distribution $d f_{0} / d t=$ $\left\langle\left\langle H f_{1}\right\rangle_{\chi}\right\rangle_{t}$. More explicitly we recover Eq. (1), with

$$
\mathcal{G}=\sum_{n} \frac{C_{\mathrm{K}} \nu / \omega\left\langle\left\langle\Re\left(H^{n} e^{i n \omega t}\right) \Re\left(h^{n} e^{i n \omega t}\right)\right\rangle_{\chi}\right\rangle_{t}}{4 \omega^{2}\left(n^{2}+\left(C_{\mathrm{K}} \nu / \omega\right)^{2}\right)},
$$

where we recall $\mathcal{G}$ is a measure of the energization from a single pump cycle. An approximate form of $\mathcal{G}$ that is easy to evaluate is given in the Methods subsection Fitting the results of $\mathcal{G}$.

We validate the analytical model of Eqs. (1) and (6) by integrating Eq. (3) numerically for a range of $v$ and considering a


Fig. 4 Comparison of $f_{e}(v)$ evolution. a The energization $\mathcal{G}$ as a function of the amplitude of a fluctuation, $\delta B / B_{0}$, and the scattering frequency, $\nu$, for the numerically-computed Lorentz (solid) and Krook (dashed) operators, as well as the analytical solution. $\mathbf{b}$ Combined estimate of the evolution of the distribution function from magnetic pumping and compressional heating for the points denoted in colored lines in Fig. 2a, obtained by integrating Eqs. (1) and (6) for $T=15 \times 2 \pi / \omega$, with $\delta B / B_{0}=0.7$, and $\nu /(\omega / 2 \pi)=0.75$. c Reproduction of Fig. 2e. The green dashed line denotes what the final distribution would look like if it only underwent compressional heating. Using these conservative parameters, $\left(T_{\text {Obs }} / T_{\text {Exp }}\right)_{\text {Theory }}=0.83\left(T_{\text {Obs }} / T_{\text {Exp }}\right)_{\mathrm{MMS}^{\prime}}$ where most of this difference stems from the discrepancy in low energy particles.

range of perturbation amplitudes, $\delta B / B_{0}$. Numerical values of $\mathcal{G}$ in Eq. (6) are estimated through Eq. (7),

$$
\omega \mathcal{G}_{\text {estimate }}=\left\langle\frac{\left(\int_{0}^{T}(\partial f / \partial t) d t\right) / T}{\frac{1}{v^{2}} \frac{\partial}{\partial v}\left(v^{4} \frac{\partial f}{\partial v}\right)}\right\rangle_{v},
$$

where $T=2 \pi / \omega$ and the numerator and denominator are found to be nearly linearly dependent functions of $v$ before averaging. In Fig. 4a, the numerical model of Eq. (3) is evaluated both with the full Lorentz operator and its Krook approximation. The analytic solution in Eq. (6) (dotted) is based on the Krook model and is in good agreement with the numerical Krook result in which the efficiency of pumping increases by a factor $\sim 100$ as $\delta B / B_{0}$ is increased from 0.3 to 0.9 .

Comparison model to spacecraft data. Using the earliest spectrum (yellow) selected in Fig. 2e with a small amount of smoothing as the initial condition, we apply the model in Eqs. (1) and (6) to predict the evolution of the pitch-angle-averaged distributions recorded by MMS. Compressional heating is modeled by including $-\dot{n} /(3 n) v(\partial f / \partial v)$ on the right-hand-side of Eq. $(1)^{6}$. The result of this calculation is shown in Fig. $4 \mathrm{~b}$ and is in good agreement with the observation from Fig. 2e, repeated for convenience in Fig. 4c. While there are some differences at lower 
energies, likely due to the effects of electric fields, which are neglected in our approach, the model provides a good account for the energization of electrons at large energies. The green dashed lines in Figs. 2e and $4 \mathrm{c}$ are the final spectra when considering compression alone, clearly underestimating the level of energization. Downstream of the shock front (for $t>11: 44: 44 \mathrm{UT}$ ) the energization rate by pumping declines with the frequency of the fluctuations, and in combination with parallel streaming losses is consistent with a drop in the fluxes of superthermal electrons.

\section{Discussion}

We have here presented an energization mechanism, magnetic pumping, that becomes applicable to superthermal particles, $v \gg \omega / k$, when the effects of trapping are retained. The MMS observations provide evidence that magnetic pumping has a significant role in electron energization in the region of the Earth's bow shock. Given the potential universal applicability of the model, this could have a far-reaching impact on our understanding of electron and superthermal ion energization in many other plasma environments where particles with $v \gg \omega / k$ are observed, such as the solar corona, cosmic ray generation pumped by magnetic turbulence in the interstellar medium, or possibly shocks driven by supernova explosions.

\section{Methods}

Finding $\boldsymbol{j}$ as a function of $\chi$. The kinetic description applied in this work is limited to the superthermal particles characterized by speeds, $v$, sufficiently large that the Lorentz force is dominated by the magnetic term, i.e., $v B \gg E$. To be more specific, in our drift kinetic analysis we are concerned with the parallel motion along the magnetic field, in general governed by forces due to the parallel electric field and the magnetic mirror force. For plasma variations of scale length $L$, the magnitude of these forces can be estimated as $\left|e \nabla_{\|} \Phi\right| \simeq T_{\mathrm{e}} / L$ and $\left|\mu \nabla_{\|} B\right| \simeq m v^{2} \delta B /\left(2 B_{0} L\right)$, and it follows that the superthermal limit requires $v^{2} \gg v_{\mathrm{t}}^{2} B_{0} /(\delta B)$, where $v_{\mathrm{t}}$ is the electron thermal speed and $\delta B / B_{0}$ is the normalized magnetic fluctuation amplitude.

In the superthermal limit the description of the orbit motion is significantly simplified as the value of $v_{\|} / v=\sqrt{1-\Lambda B}$ along an orbit is only dependent on $\Lambda=\mu B_{0} / \mathcal{E}$ (and independent of the electron energy $\mathcal{E}$ with the assumption $E_{\|}=$ 0 ). This strongly simplifies the calculation of the second adiabatic invariant $J(v, \Lambda)$ because $j=J /(4 v L)$ is then a function of only $\Lambda$, readily evaluated numerically for the slowly evolving magnetic perturbation considered, as illustrated in Fig. 5, where

$$
j(\Lambda, t)=\frac{1}{4 L} \oint \frac{v_{\|}}{v} d l=\frac{1}{2 L} \int_{0}^{L_{\mathrm{b}}} \sqrt{1-\Lambda B(t, x)} d x .
$$

Given this calculation of $j(\Lambda, t)$ over the course of a full pump cycle we can determine $\Lambda(\chi, t)$ and in turn obtain $j(\chi, t)$ as shown in Fig. $5 \mathrm{c}$. This function is fundamental to our analysis as it is related to the instantaneous rate of particle energization. Because $d J / d t=d(v j) / d t=0$ it follows that $j d v / d t+v d j / d t=0$. Furthermore, as $j=j(\chi, t)$ and $d \chi / d t=0$, we have $d j / d t=\partial j /\left.\partial t\right|_{\chi}$, such that $v^{-1} d v / d t=-j^{-1} \partial j /\left.\partial t\right|_{\chi}$. We then obtain the result implicit in Eq. (3) that

$$
\left.\frac{d \mathcal{E}}{d t}\right|_{\chi}=-\mathcal{E} H, \quad \text { where }\left.H \equiv \frac{2}{j} \frac{\partial j}{\partial t}\right|_{\chi} .
$$

Particle conservation in $\langle(\ldots)\rangle_{\chi}$. Without loss of generality, in our analysis magnetic-field lines in the $(x, z)$-plane are characterized by the flux function $\Psi$, and we consider a flux-tube with constant width $\Delta y$ in the $y$ direction. The width in the $z$ direction varies as $1 / B$ and is parameterized by $\Delta \Psi$. The total number of particles within our flux tube of length $L$ must be conserved, i.e.,

$$
N=\int f d^{3} v d^{3} x=\frac{2 \pi \Delta y}{m^{2}} \int d \mathcal{E} \int d \mu \int d \Psi \tau_{\mathrm{b}} f .
$$

For the point $x=L / 2, B=B_{0}$ we can evaluate $\Delta \Psi=B_{0} \Delta z$, where $\Delta z$ is the width of the flux tube at that $x$ location. Furthermore, because $\tilde{\tau}_{\mathrm{b}}=v \tau_{\mathrm{b}} /(4 L)$ and $d \mathcal{E} d \mu=m^{2} v^{3} d v d \Lambda / B_{0}$ we get

$$
N=2 \pi \Delta y \Delta z L \int v^{2} d v \int d \Lambda \tilde{\tau}_{\mathrm{b}} f .
$$

Rewriting this in terms of our preferred variables, $(v, \chi)$ the expression becomes

$$
N=2 \pi \Delta y \Delta z L \int v^{2} d v \int d \chi\left(\frac{d \Lambda}{d \chi}\right) \tilde{\tau}_{\mathrm{b}} f
$$
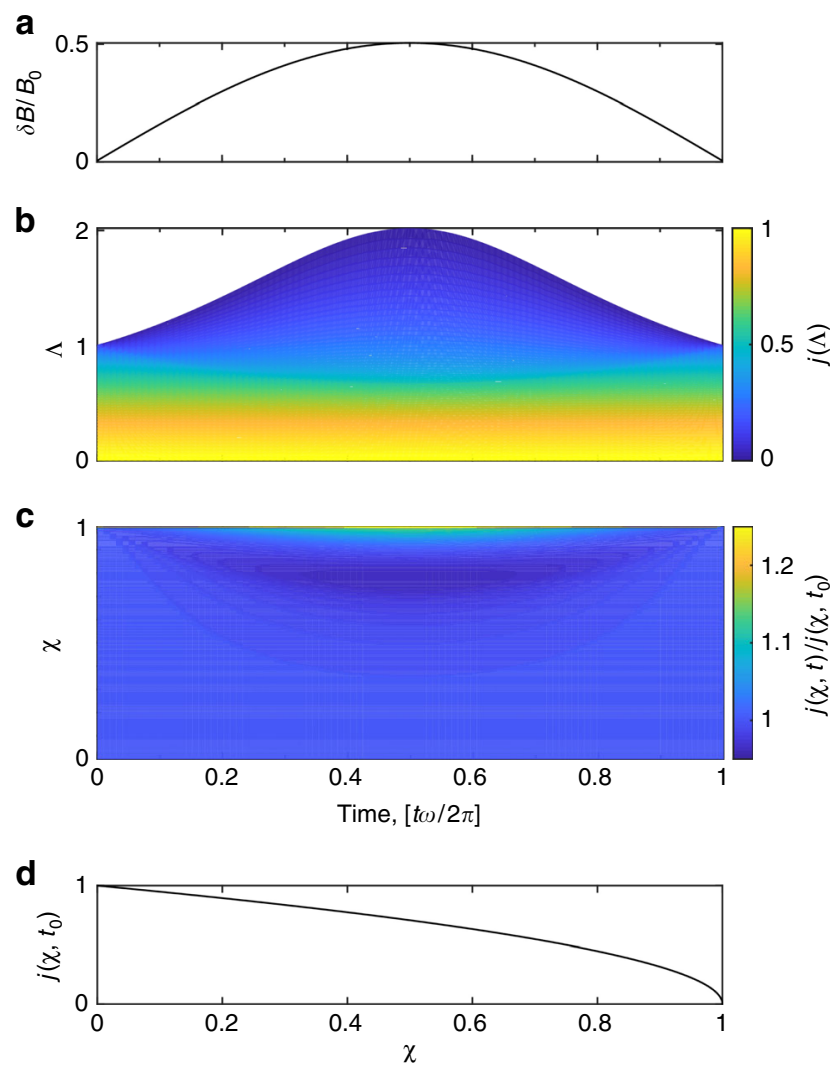

Fig. $\mathbf{5} \boldsymbol{j}$ as a function of invariants. a Plot of the maximum magnetic-field amplitude over the course of a fluctuation for a maximum magnetic field of $\delta B / B_{0}=0.5$. $\mathbf{b}$ Plot of $j$ as a function of $\Lambda$ over the course of a fluctuation for the magnetic field in a. c Plot of $j(\chi, t)$ normalized by $j\left(\chi, t_{0}\right)$ for the same fluctuation. d Plot of $j\left(\chi, t_{0}\right)$.

This motivates the averaging operator

$$
\langle(\ldots)\rangle_{\chi}=\frac{\int d \chi\left(\frac{d \Lambda}{d \chi}\right) \tilde{\tau}_{\mathrm{b}}(\ldots)}{\int d \chi\left(\frac{d \Lambda}{d \chi}\right) \tilde{\tau}_{\mathrm{b}}}
$$

such that the pitch-angle averaged distribution becomes

$$
F(v)=\langle f\rangle_{\chi} .
$$

In turn, the total number of particles can then be written as

$$
N=2 \pi \Delta y \Delta z L\left(\int d \chi\left(\frac{d \Lambda}{d \chi}\right) \tilde{\tau}_{\mathrm{b}}\right) \int F(v) v^{2} d v
$$

From the form of Eq. (13) it is clear that $F v^{2} d v$ is proportional to the number of particles in the flux tube within a differential speed interval, $d v$.

Obtaining an estimate for the effective scattering frequency. We can estimate how much scattering is needed to match the spacecraft observations by comparing the MMS distributions to theoretical distributions generated by integrating Eq. (3) for a range of scattering frequencies, $v$. By comparing the anisotropic features in the MMS distribution to the features in this set of theoretical distribution functions, we can estimate the effective scattering frequency. Based on a least-squared-fit analysis, the scattering frequency that best fit this data varies as a function of velocity, where $v /(\omega / 2 \pi) \in[0.25,1.5]$. A scattering frequency within this range, $v /(\omega / 2 \pi)=0.75$, is chosen to generate a comparison with the MMS data, where the resulting scattered distribution is shown in Fig. 3(k). A more thorough investigation into how the estimated scattering frequency varies as a function of velocity can be found in ref. ${ }^{33}$, while examples of theoretical distributions scattered at a set of different rates are shown in Fig. 6.

Observations of distributions throughout the encounter. In Fig. 3 of the main text we demonstrated anisotropic distribution with features matching those of the numerical model. To further demonstrate that these type of anisotropic distributions are representative for the full event, we here include in Fig. 7 electron 
distribution functions measured by MMS along the entire bow shock crossing. Compared to the peak and valley distributions in Fig. 3 these follow the expectations from the theory.

Fitting the results of $\mathcal{G}$. For an easy-to-evaluate approximation of the theory, the results of Eq. (6) can be fit as

$$
\mathcal{G} \simeq 0.07\left(\left(\frac{\delta B}{B_{0}}\right)^{2.6}+3\left(\frac{\delta B}{B_{0}}\right)^{5.6}\right) \frac{C_{\mathrm{K}} \nu / \omega}{2.3+C_{\mathrm{K}}^{2} \nu^{2} / \omega^{2}},
$$

where $C_{\mathrm{K}}=1.15 /\left(\delta B / B_{0}\right)^{1.13}$, as discussed in the text and the last part of this methods section. This fitting, plotted alongside the results from Fig. $4 \mathrm{a}$ are plotted


Fig. 6 Estimating the effective scattering frequency. a-d Electron distribution functions computed using Eq. (3) integrated at $\left(\delta B / B_{0}\right)$ $\sin \left(\omega t_{0}\right)=0.5$ for a range of scattering frequencies. e Plot of the magnetic field for the relevant bow shock crossing. $\mathbf{f}$ MMS3 observation of an electron distribution function at the orange line in $\mathbf{e}$, where we estimate $\tilde{B}=0.5$. in Fig. 8. We note that this approximation for $\mathcal{G}$ is also valid in the limit of $\delta B / B_{0} \rightarrow 0$

Orbit averaging of the Lorentz operator. As outlined above, our model is averaged over the fast electron orbit motion. Locally we assume that the scattering process is described by the familiar Lorentz pitch-angle scattering operator, $\mathcal{L}$, which is then subject to orbit averaging 20,29 . For this orbit averaging, it is convenient to express $\mathcal{L}$ in terms of the constant of motion variable $\Lambda$. Starting with the Lorentz pitch-angle scattering operator in terms of $\xi=v_{\|} / v$,

$$
\mathcal{L}=\frac{1}{2} \frac{\partial}{\partial \xi}\left(1-\xi^{2}\right) \frac{\partial}{\partial \xi}
$$

we rewrite the scattering operator in terms of the new variables, $\Lambda$ and $\mathcal{E}$

$$
\mathcal{L}=\left.\left.\frac{m v_{\|} B_{0}^{2}}{\mathcal{E}^{2} B} \frac{\partial}{\partial \Lambda}\right|_{\mathcal{E}} ^{\mu v_{\|}} \frac{\partial}{\partial \Lambda}\right|_{\mathcal{E}}, \quad v_{\|}=\sqrt{2 \mathcal{E}(1-\Lambda \tilde{B}) / m}
$$

which then takes the form

$$
\mathcal{L}=\left.\left(\frac{2}{\tilde{B}}-3 \Lambda\right) \frac{\partial}{\partial \Lambda}\right|_{\mathcal{E}}+\left.\frac{2}{\tilde{B}} \Lambda(1-\Lambda \tilde{B}) \frac{\partial^{2}}{\partial \Lambda^{2}}\right|_{\mathcal{E}}
$$

After averaging along the spatial dimension of the flux tube we then obtain the orbit-averaged operator,

$$
\begin{aligned}
\langle\mathcal{L}\rangle_{x}= & \left.\left(2\left\langle\frac{1}{B}\right\rangle_{x}-3 \Lambda\right)_{\frac{\partial}{\partial \Lambda}}\right|_{\mathcal{E}} \\
& +\left.2 \Lambda\left(\left\langle\frac{1}{B}\right\rangle_{x}-\Lambda\right)_{\frac{\partial^{2}}{\partial \Lambda^{2}}}\right|_{\mathcal{E}}
\end{aligned}
$$

where $\langle(\ldots)\rangle_{x}=1 /\left(\tilde{\tau}_{\mathrm{b}} L\right) \int_{0}^{L_{\mathrm{b}}} d x(\ldots) / \sqrt{1-\Lambda\left(B / B_{0}\right)}$ is the integral over the orbits, where $\tilde{\tau}_{\mathrm{b}}=v \tau_{\mathrm{b}} /(4 L)$ is the speed-normalized bounce time.

Calibrating the Krook operator as a function of $\delta B / B_{0}$. As is clear from the form of Eq. (14), the Lorentz operator describes diffusion of anisotropic features of the distribution function, $f$. Accordingly, the diffusion time scales as $\tau_{\mathrm{D}} \propto(\delta \xi)^{2}$, where $\delta \xi$ is the typical scale for the anisotropic features of $f$. As the magnetic field increases, the trapped portion of the distribution function will increase commensurately, yielding larger values of $\delta \xi$.

The Krook operator does not have this same dependence on $\delta \xi$, as the rate of isotropization is in fact independent of $\delta \xi$. When computing the Krook collision operator the new, scattered distribution function is formed from a linear combination of the original distribution function, and a fully isotropized version of

a
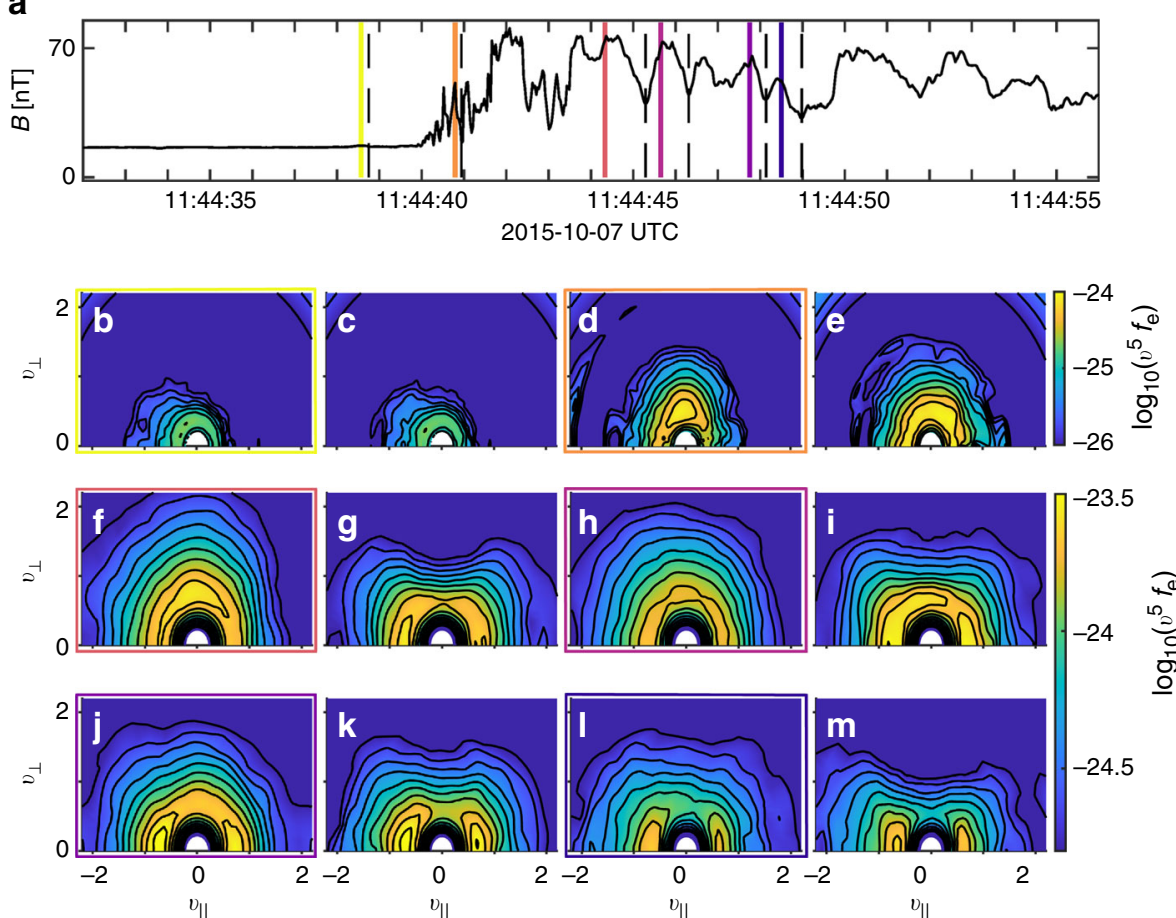

Fig. 7 Distributions along the bow shock crossing. a Magnetic field observed by MMS4 along the bow shock crossing. b-m Electron distribution functions at the peaks and the corresponding valleys of magnetic fluctuations along the bow shock crossing are plotted. The distributions with the colored borders are the distributions taken at the magnetic peaks at the times denoted by the colored lines in $\mathbf{a}$. The adjacent distributions are taken at the associated valleys denoted by the adjacent dashed lines in $\mathbf{a}$. 


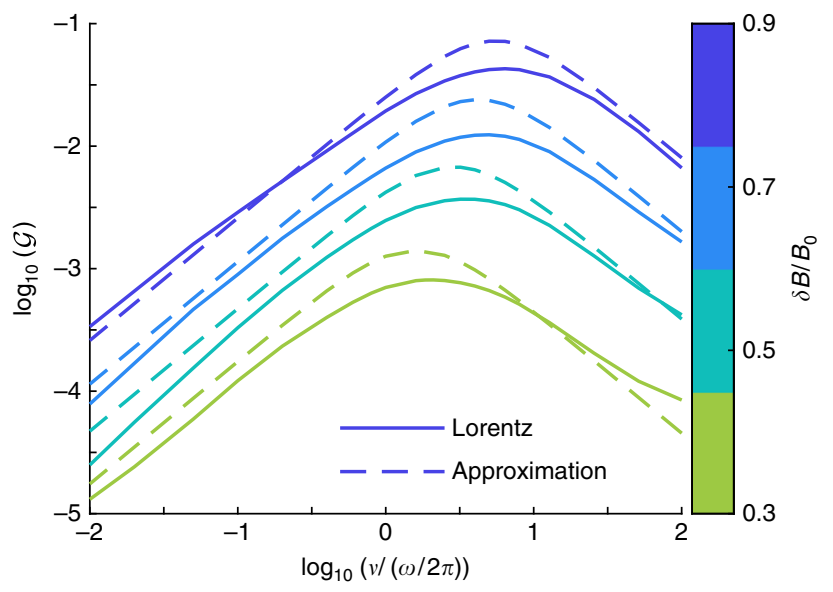

Fig. 8 Fitting the results of $\mathcal{G}$. Reproduction of Lorentz distributions generated in Fig. 4 along with the results from the approximate form of $\mathcal{G}$. the distribution function,

$$
f_{t+\Delta t}=\alpha f_{t}+(1-\alpha)\left\langle f_{t}\right\rangle_{\chi},
$$

where $\alpha=\exp \left(-\nu C_{\mathrm{K}} \Delta t\right)$ determines the rate of isotropization.

To approximate the $\delta \xi$ dependency of $\mathcal{L}$ we have introduced the coefficient $C_{\mathrm{K}}$. From an analysis of the kinetic equation it follows that $\delta \xi$ of $f$ is similar to $\delta \xi$ of $g=j^{2}$ $+\Lambda$, which we used to provide a calibration for the efficiency of the Krook operator:

$$
C_{\mathrm{K}}=\frac{\left\langle\left(g-\langle g\rangle_{\Lambda}\right) \mathcal{L} g\right\rangle_{\Lambda}}{\left\langle\left(g-\langle g\rangle_{\Lambda}\right)^{2}\right\rangle_{\Lambda}} .
$$

By repeating this computation for a multiple $\delta B / B_{0}$ we find the result scales as

$$
C_{\mathrm{K}}\left(\delta B / B_{0}\right)=1.15 /\left(\delta B / B_{0}\right)^{1.13}
$$

which is the result we used to compute the Krook distribution curves earlier in the paper.

\section{Data availability}

All relevant data are available from the corresponding author upon reasonable request. Additionally, the MMS spacecraft observations used in the production of this work can be found in the MMS Science Data Center (https://lasp.colorado.edu/mms/sdc/public/ datasets/fpi/ and https://www.lasp.colorado.edu/mms/sdc/public/datasets/fields/).

\section{Code availability}

All relevant code and Matlab routines are available from the corresponding author upon reasonable request.

Received: 18 December 2019; Accepted: 14 May 2020;

Published online: 10 June 2020

\section{References}

1. Oka, M. et al. Electron power-law spectra in solar and space plasmas. Space Sci. Rev. 214, 82 (2018).

2. Fisk, L. A. \& Gloeckler, G. The common spectrum for accelerated ions in the quiet-time solar wind. Astrophys. J. 640, L79-L82 (2006).

3. WilsonIII, L. B. et al. Quantified energy dissipation rates in the terrestrial bow shock: 2. Waves and dissipation. J. Geophys. Research Space Phys. 119, 6475-6495 (2014).

4. Alexandrov, A. F., Bogdankeich, L. S. \& Rukhadze, A. A.Principles of Plasma Electrodynamics (Springer, 1984).

5. Voitenko, Y. \& Pierrard, V. Velocity-space proton diffusion in the solar wind turbulence. Sol. Phys. 288, 369-387 (2013).

6. Lichko, E., Egedal, J., Daughton, W. \& Kasper, J. Magnetic pumping as a source of particle heating and power-law distributions in the solar wind. Astrophys. J. Lett. 850, L28 (2017).

7. Blandford, R. \& Eichler, D. Particle-acceleration at astrophysical shocks-a theory of cosmic-ray origin. Phys. Rep.-Rev. Sect. Phys. Lett. 154, 1-75 (1987).

8. Oka, M. et al. Electron scattering by low-frequency Whistler waves at Earth's bow shock. Astrophys. J. 886, 53-63 (2019).
9. Katou, T. \& Amano, T. Theory of stochastic shock drift acceleration for electrons in the shock transition region. Astrophys. J. 874, 119-130 (2019).

10. Amano, T. et al. Observational evidence for stochastic shock drift acceleration of electrons at the Earth's bow shock. Phys. Rev. Lett. 124, 065101 (2020).

11. Levinson, A. Electron injection in collisionless shocks. Astrophys. J. 401, 73-80 (1992).

12. Gosling, J. T., Thomson, M. F., Bame, S. J. \& Russell, C. T. Suprathermal electrons at Earth's bow shock. J. Geophys. Res. 94, 10011-10025 (1989).

13. Chew, G. F., Goldberger, M. L. \& Low, F. E. The Boltzmann equation and the one-fluid hydromagnetic equations in the absence of particle collisions. Proc. R. Soc. Lond. Ser. A 236, 112-118 (1956).

14. Oka, M. et al. Electron scattering by high-frequency Whistler waves at Earth's Bow Shock. Astrophys. J. Lett. 842, L11 (2017).

15. Howes, G. G. et al. Kinetic simulations of magnetized turbulence in astrophysical plasmas. Phys. Rev. Lett. 100, 065004 (2008).

16. Howes, G. G. et al. Gyrokinetic simulations of solar wind turbulence from ion to electron scales. Phys. Rev. Lett. 107, 035004 (2011).

17. Chandran, B. D. G. \& Hollweg, J. V. Alfvén wave reflection and turbulent heating in the solar wind from 1 solar radius to $1 \mathrm{AU}$ : an analytical treatment. Astrophys. J. 707, 1659-1667 (2009).

18. Barnes, A. Collisionless damping of hydromagnetic waves. Phys. Fluids 9, 1483 (1966).

19. Stix, T. H. Waves in Plasmas. (Springer-Verlag, New York, Inc., 1992).

20. Davidson, R. C. Methods in Nonlinear Plasma Theory (Academic Press, 1972).

21. Burch, J. L., Moore, T. E., Torbert, R. B. \& Giles, B. L. Magnetospheric multiscale overview and science objectives. Space Sci. Rev. 199, 5-21 (2016).

22. Guo, F. \& Giacalone, J. The effect of large-scale magnetic turbulence on the acceleration of electrons by perpendicular collisionless shocks. Astrophys. J. 715, 406-411 (2010).

23. Lowe, R. E. \& Burgess, D. The properties and causes of rippling in quasiperpendicular collisionless shock fronts. Ann. Geophys. Eur. Geosci. Union 21, 671-679 (2003).

24. Burgess, D. Simulations of electron acceleration at collisionless shocks: the effects of surface fluctuations. Astropys. J. 653, 316-324 (2006).

25. Morse, D. L., Destler, W. W. \& Auer, P. L. Nonstationary behavior of collisionless shocks. Phys. Rev. Lett. 28, 13-16 (1972).

26. Krasnoselskikh, V. V., Lembége, B., Savoini, P. \& Lobzin, V. V. Nonstationarity of strong collisionless quasiperpendicular shocks: theory and full particle numerical simulations. Phys. Plasmas 9, 1192 (2002).

27. Johlander, A. et al. Rippled quasiperpendicular shock observed by the magnetospheric multiscale spacecraft. Phys. Rev. Lett. 117, 165101 (2016)

28. Chen, L. J. et al. Electron bulk acceleration and thermalization at Earth's quasiperpendicular bow shock. Phys. Rev. Lett. 120, 225101 (2018).

29. Cordey, J. G. Effects of particle trapping on the slowing-down of fast ions in a toroidal plasma. Nucl. Fusion 16, 499-507 (1976).

30. Kulsrud, R. M. in Handbook of Plasma Physics (eds Rosenbluth, M. N. \& Sagdeev, R. Z.) Vol. 1 Ch. 1, 115-145, (North-Holland Publishing Company, New York, 1983).

31. Montag, P., Egedal, J., Lichko, E. \& Wetherton, B. Impact of compressibility and a guide field on Fermi acceleration during magnetic island coalescence. Phys. Plasmas 24, 062906 (2017).

32. Egedal, J., Monkhorst, H., Lichko, E. \& Montag, P. Theory of ion dynamics and heating by magnetic pumping in FRC plasma. Phys. Plasmas 25, 072510 (2018).

33. Lichko, E. Magnetic pumping as a source of particle heating. Ph.D. thesis, University of Wisconsin-Madison (2020).

34. Tsurutani, B. T. \& Rodriguez, P. Upstream waves and particles: an overview of ISEE results. J. Geophys. Res. 86, 4317 (1981).

35. Taylor, G. I. The spectrum of turbulence. Proc. R. Soc. 164, 0476-0490 (1938),

\section{Acknowledgements}

This work was supported by NASA Grant No. NNX15AJ73G. Contributions from E.L. were also supported by the Department of Defense (DoD) through the National Defense Science \& Engineering Graduate Fellowship (NDSEG) Program and the NASA Earth and Space Science Fellowship. Simulations were performed with LANL institutional computing resources.

\section{Author contributions}

This work was performed by E.L. and J.E.

\section{Competing interests}

The authors declare no competing interests. 


\section{Additional information}

Supplementary information is available for this paper at https://doi.org/10.1038/s41467020-16660-4

Correspondence and requests for materials should be addressed to E.L.

Peer review information Nature Communications thanks Jason TenBarge, and other, anonymous, reviewer(s) for their contribution to the peer review of this work. Peer reviewer reports are available.

Reprints and permission information is available at http://www.nature.com/reprints

Publisher's note Springer Nature remains neutral with regard to jurisdictional claims in published maps and institutional affiliations. (c) (i) Open Access This article is licensed under a Creative Commons Attribution 4.0 International License, which permits use, sharing, adaptation, distribution and reproduction in any medium or format, as long as you give appropriate credit to the original author(s) and the source, provide a link to the Creative Commons license, and indicate if changes were made. The images or other third party material in this article are included in the article's Creative Commons license, unless indicated otherwise in a credit line to the material. If material is not included in the article's Creative Commons license and your intended use is not permitted by statutory regulation or exceeds the permitted use, you will need to obtain permission directly from the copyright holder. To view a copy of this license, visit http://creativecommons.org/ licenses/by/4.0/.

(C) The Author(s) 2020 